\title{
On the quadratic stability of switched interval systems: Preliminary results
}

\author{
Ezra Zeheb, Oliver Mason, Selim Solmaz and Robert Shorten
}

\begin{abstract}
In this paper we present some preliminary results on the quadratic stability of switched systems with uncertain parameters. We show that the quadratic stability of a class of switched uncertain systems may be readily verified using simple algebraic conditions. Examples are presented to demonstrate the efficacy of our techniques.
\end{abstract}

\section{INTRODUCTION}

Recent years have witnessed great interest in the mathematics, computer science, and control engineering communities in the analysis and design of hybrid dynamic systems. Roughly speaking, hybrid systems are systems whose behaviour is described mathematically using a mixture of classical differential/difference equations and logic based switching. Examples of such systems are pervasive in engineering practice and can be readily found in the aircraft, automotive and communications industries. Despite the recent interest in these systems, their behaviour is not yet completely understood. Given the pervasive nature of such systems, in particular in safety critical applications, understanding their stability properties is one of the most pressing issues that needs to be addressed.

In this paper we consider the most widely studied hybrid dynamical system; namely hybrid systems constructed by switching between a set of linear vector fields. The problem of determining the stability properties of such systems is of great importance in many fields of applied and theoretical research: see [1], [2] and the references therein for an overview of some of the historical work carried out in the mathematics, control engineering and computer science communities. One method of establishing the exponential stability of a dynamical system $\Sigma: \dot{x}(t)=A(t) x(t)$ (where $x(t) \in \mathbb{R}^{n}, A(t) \in \mathbb{R}^{n \times n}, A(t) \in\left\{A_{1}, \ldots, A_{m}\right\}$ ) is to show that for some positive definite matrix $P$ the quadratic Lyapunov function $x(t)^{T} P x(t)$ is decreasing in time; namely that $A_{i}^{T} P+P A_{i}<0$ for all $1 \leq i \leq m$. When such a function exists, then the associated LTI systems

$$
\Sigma_{A_{i}}: \dot{x}(t)=A_{i} x(t) \quad 1 \leq i \leq m
$$

are said to have a common quadratic Lyapunov function (CQLF). Recently, motivated by the stability of switched systems [3], and robust control problems [4], the problem

E. Zeheb is with the Technion, Haifa, Israel zeheb@ee.technion.ac.il (joint first author)

O. Mason is with the Hamilton Institute, NUI Maynooth, Co. Kildare, Ireland oliver.mason@may. ie (joint first author)

R. Shorten is with the Hamilton Institute, NUI Maynooth, Co. Kildare, Ireland robert. shorten@may . ie

S. Solmaz is with the Hamilton Institute, NUI Maynooth, Co. Kildare, Ireland selim.solmaz@may.ie of determining compact conditions for the existence of a CQLF for a finite number of LTI systems has assumed a position of great theoretical importance in the mathematics and engineering communities; see [5], [6], [7], [8], [9], [10], [11], [12], [13], [14], [15], [16], [17], [18], [19], [20], [21], [22], [23], [24], [25] for some of the most important historical and recent work in this area as well as the recent paper [25] for the most complete solution to this problem to-date. Notwithstanding the progress that has been made on the general CQLF existence problem, the problem of determining whether or not a set of LTI systems subject to interval uncertainty has a CQLF has received relatively little attention, despite its obvious considerable practical importance. Our objective in this paper is to study this problem for a restricted class of switching systems subject to interval uncertainty; namely the class of switching systems given by $\dot{x}=A(t) x, A(t) \in\left\{A, A-g h^{T}\right\}$, where $g, h \in \mathbb{R}^{n}$, and the system matrices $A, A-g h^{T}$ are subject to interval uncertainty of the form $\underline{a}_{i j} \leq a_{i j} \leq \bar{a}_{i j}$.

\section{PREliminary RESUlts}

Throughout this note $\mathbb{R}$ and $\mathbb{C}$ denote the fields of real and complex numbers respectively. We denote $n$-dimensional real Euclidean space by $\mathbb{R}^{n}$ and the space of $n \times n$ matrices with real entries by $\mathbb{R}^{n \times n}$. For a vector $x$ in $\mathbb{R}^{n}$, $x_{i}$ denotes the $i^{\text {th }}$ component of $x$ and for $A$ in $\mathbb{R}^{n \times n}$, we denote the entry in the $(i, j)$ position by $a_{i j}$. In this section, we introduce a number of concepts and preliminary mathematical results that shall be needed in the remainder of the paper.

\section{CQLF existence for systems differing by rank one:}

To begin with, we recall a number of results concerned with the problem of CQLF existence for pairs of fixed coefficient LTI systems whose system matrices differ by rank one.

The classical single-input single-output (SISO) Circle Criterion [26] gave a necessary and sufficient condition for two fixed coefficient LTI systems with system matrices in companion form to have a CQLF. Formally, if $A, A-g h^{T}$ are two Hurwitz matrices in $\mathbb{R}^{n \times n}$ in companion form, then the LTI systems $\Sigma_{A}, \Sigma_{A-g h^{T}}$ have a CQLF if and only if the rational function

$$
1+h^{T}(s I-A)^{-1} g
$$

is strictly positive real (SPR), meaning that

$$
1+\operatorname{Re}\left\{h^{T}(j \omega I-A)^{-1} g\right\}>0
$$


for all $\omega$ in $\mathbb{R}$. Moreover, it follows from Meyer's extension of the KYP Lemma [27] that the condition (2) is also sufficient for CQLF existence for two LTI systems $\Sigma_{A}$, $\Sigma_{A-g h^{T}}$ where $A, A-g h^{T}$ are Hurwitz matrices differing by rank one, but not necessarily in companion form. Recently in [7], [22], it has been established that the frequency domain condition (2) is equivalent to a simple condition on the eigenvalues of the matrix product $A\left(A-g h^{T}\right)$. This equivalence was first demonstrated in [7] for matrices in companion form and then extended to the case of a general pair of Hurwitz matrices $A_{1}, A_{2}$ with $\operatorname{rank}\left(A_{2}-A_{1}\right)=1$ in [22].

Theorem 2.1: Let $A, A-g h^{T}$ be Hurwitz matrices in $\mathbb{R}^{n \times n}$, where $g, h \in \mathbb{R}^{n}$. Then

$$
1+\operatorname{Re}\left\{h^{T}(j \omega I-A)^{-1} g\right\}>0 \text { for all } \omega \in \mathbb{R}
$$

if and only if the matrix product $A\left(A-g h^{T}\right)$ has no negative real eigenvalues.

For completeness, the proof of this result is included in the appendix. Also, note that in the paper [28], a number of implications of the above equivalence for multiplier-type stability results were described.

Combining the result of Theorem 2.1 with Meyer's extension of the KYP Lemma [27], yields the following timedomain condition for CQLF existence for systems with system matrices differing by rank one.

Theorem 2.2: [22] Let $A, A-g h^{T}$ be two Hurwitz matrices in $\mathbb{R}^{n \times n}$ where $g, h$ are vectors in $\mathbb{R}^{n}$. A necessary and sufficient condition for the existence of a common quadratic Lyapunov function for the LTI systems, $\Sigma_{A}$, and $\Sigma_{A-g h^{T}}$ is that the matrix product $A\left(A-g h^{T}\right)$ does not have any negative real eigenvalues.

In the remainder of this paper, our primary concern shall be with illustrating how Theorem 2.2 may be used to obtain results on CQLF existence for pairs of LTI systems with interval uncertainty in the entries of their system matrices.

\section{Kharitonov's theorem and rational transfer functions:}

In the next section, we shall derive two closely related results on CQLF existence for systems $\Sigma_{A}, \Sigma_{A-g h^{T}}$ whose coefficients are subject to interval uncertainty. The first of these is based on a result derived in [29] that extended Kharitonov's Theorem on the stability of interval polynomials to the case of proper rational functions. In order to fix our notation, consider the family of interval polynomials $\mathcal{P}$ given by

$$
p(s)=p_{0}+p_{1} s+\cdots+p_{n} s^{n},
$$

where $\underline{p}_{i} \leq p_{i} \leq \bar{p}_{i}$ for $1 \leq i \leq n$. Then Kharitonov's Theorem states that in order to check that the roots of all polynomials in the family $\mathcal{P}$ are in the open left half of the complex plane it suffices to check this for the four
Kharitonov polynomials

$$
\begin{aligned}
k_{1}^{\mathcal{P}}(s) & =\underline{p}_{0}+\underline{p}_{1} s+\bar{p}_{2} s^{2}+\bar{p}_{3} s^{3}+\cdots \\
k_{2}^{\mathcal{P}}(s) & =\underline{p}_{0}+\bar{p}_{1} s+\bar{p}_{2} s^{2}+\underline{p}_{3} s^{3}+\underline{p}_{4} s^{4} \\
k_{3}^{\mathcal{P}}(s) & =\bar{p}_{0}+\underline{p}_{1} s+\underline{p}_{2} s^{2}+\bar{p}_{3} s^{3}+\bar{p}_{4} s^{4}+\cdots \\
k_{4}^{\mathcal{P}}(s) & =\bar{p}_{0}+\bar{p}_{1} s+\underline{p}_{2} s^{2}+\underline{p}_{3} s^{3}+\cdots
\end{aligned}
$$

In the paper [29], this result was extended in the following way. Let $\mathcal{P}, \mathcal{Q}$ be two families of interval polynomials given by

$$
\begin{aligned}
\mathcal{P}= & \left\{p_{0}+p_{1} s+\cdots+p_{n} s^{n}:\right. \\
& \left.\underline{p}_{i} \leq p_{i} \leq \bar{p}_{i}, \text { for } 1 \leq i \leq n\right\} \\
\mathcal{Q}= & \left\{q_{0}+q_{1} s+\cdots+q_{m} s^{m}:\right. \\
& \left.\underline{q}_{i} \leq q_{i} \leq \bar{q}_{i}, \text { for } 1 \leq i \leq m\right\},
\end{aligned}
$$

where $n \leq m$. Then consider the family $\mathcal{P} / \mathcal{Q}$ of proper rational functions of the form

$$
\frac{p(s)}{q(s)}
$$

where the polynomials $p$ and $q$ belong to $\mathcal{P}$ and $\mathcal{Q}$ respectively. We shall make use of the following result from [29] about the strict positive realness of all of the rational functions in $\mathcal{P} / \mathcal{Q}$ in the next section.

Theorem 2.3: Every transfer function in the family $\mathcal{P} / \mathcal{Q}$ is strictly positive real if and only if the following eight transfer functions are strictly positive real.

$$
\begin{aligned}
& \frac{k_{2}^{\mathcal{P}}(s)}{k_{1}^{\mathcal{Q}}(s)}, \frac{k_{3}^{\mathcal{P}}(s)}{k_{1}^{\mathcal{Q}}(s)}, \frac{k_{1}^{\mathcal{P}}(s)}{k_{2}^{\mathcal{Q}}(s)}, \frac{k_{4}^{\mathcal{P}}(s)}{k_{2}^{\mathcal{Q}}(s)}, \\
& \frac{k_{1}^{\mathcal{P}}(s)}{k_{3}^{\mathcal{Q}}(s)}, \frac{k_{4}^{\mathcal{P}}(s)}{k_{3}^{\mathcal{Q}}(s)}, \frac{k_{2}^{\mathcal{P}}(s)}{k_{4}^{\mathcal{Q}}(s)}, \frac{k_{3}^{\mathcal{P}}(s)}{k_{4}^{\mathcal{Q}}(s)},
\end{aligned}
$$

where $k_{i}^{\mathcal{P}}, k_{i}^{\mathcal{Q}}, 1 \leq i \leq 4$, are the Kharitonov polynomials corresponding to the interval polynomial families $\mathcal{P}$ and $\mathcal{Q}$ respectively.

\section{Positivity of multi-variable polynomials:}

Finally for this section, we present the following theorem from [30] on the positivity of multi-variable polynomials. Later, we shall combine this result with Theorem 2.2 to obtain necessary and sufficient conditions for CQLF existence for certain classes of systems subject to interval uncertainty. Theorem 2.4 below provides necessary and sufficient conditions for a polynomial $p(x)$ of $n$-variables (defined for $x \in \mathbb{R}^{n}$ ) to be positive for all $x$ in a region $D^{n}$ of the form

$$
\left\{x \in \mathbb{R}^{n}: \underline{x}_{i} \leq x_{i} \leq \bar{x}_{i}\right\},
$$

where the end-points $\underline{x}_{i}, \bar{x}_{i}$, may be finite or infinite.

Theorem 2.4: [30] Let $p(x)$ be a real-coefficient polynomial of $n$-variables, and $D^{n}$ be a region in $\mathbb{R}^{n}$ of the form (11). Then $p(x)>0$ for all $x \in D^{n}$ if and only if: 
(i) the polynomials obtained by setting the value of one of the variables $x_{i}$ to one of the end-points $\underline{x}_{i}$ or $\bar{x}_{i}$ are all positive for the permitted values of the remaining variables $x_{1}, \ldots, x_{i-1}, x_{i+1}, \ldots, x_{n}$;

(ii) there is no $x \in D^{n}$ satisfying the $n$ equations

$$
\begin{aligned}
p(x) & =0 \\
\frac{\partial p}{\partial x_{i}}(x) & =0 \quad \text { for } 1 \leq i \leq n-1 .
\end{aligned}
$$

\section{MAIN RESULTS}

\section{A. CQLF existence for interval matrices in companion form}

In this subsection, we derive a result on CQLF existence for a pair of interval matrix families in companion form. More formally, consider the matrix families $\mathcal{A}, \mathcal{B}$ consisting of matrices of the form

$$
\begin{aligned}
A= & \left(\begin{array}{llrrr}
0 & 1 & 0 & \ldots & 0 \\
0 & 0 & 1 & \ldots & 0 \\
\vdots & & & & \\
0 & 0 & 0 & \ldots & 1 \\
-a_{0} & -a_{1} & -a_{2} & \ldots & -a_{n-1}
\end{array}\right), \\
B= & \left(\begin{array}{llllr}
0 & 1 & 0 & \ldots & 0 \\
0 & 0 & 1 & \ldots & 0 \\
\vdots & & & & \\
0 & 0 & 0 & \ldots & 1 \\
-b_{0} & -b_{1} & -b_{2} & \ldots & -b_{n-1}
\end{array}\right),
\end{aligned}
$$

where $\underline{a}_{i} \leq a_{i} \leq \bar{a}_{i}, \underline{b}_{i} \leq b_{i} \leq \bar{b}_{i}$ for $0 \leq i \leq n-1$. In Theorem 3.1 below, we consider the following problem.

Determine necessary and sufficient conditions for any pair of LTI systems $\Sigma_{A}, \Sigma_{B}$ with $A \in \mathcal{A}$, $B \in \mathcal{B}$ to have a CQLF.

In the context of this problem, the following brief points should be noted.

(i) In order for the LTI systems $\Sigma_{A}, \Sigma_{B}$ to have a CQLF, it is necessary that the systems are both stable, or equivalently that their system matrices, $A, B$ are Hurwitz. As our concern here is with the question of CQLF existence for pairs $\Sigma_{A}, \Sigma_{B}$ with $A \in \mathcal{A}, B \in \mathcal{B}$, we shall assume that all of the matrices belonging to the families $\mathcal{A}, \mathcal{B}$ are Hurwitz.

(ii) The problem of determining whether or not a family of interval matrices consists entirely of Hurwitz matrices has itself been the subject of a considerable amount of research, and several results have been published providing methods for testing such families for stability [31], [32], [33]. Note also that in the case of interval matrices in companion form, Kharitonov's Theorem can be used to test for stability.

For notational convenience, we shall denote the companion matrix whose last row is $\left(-a_{0},-a_{1}, \ldots,-a_{n-1}\right)$ by
$C\left(a_{0}, \ldots, a_{n-1}\right)$. Then for the interval matrix family $\mathcal{A}$, we can construct the four matrices

$$
\begin{aligned}
& A_{1}=C\left(\underline{a}_{0}, \underline{a}_{1}, \bar{a}_{2}, \bar{a}_{3}, \ldots\right) \\
& A_{2}=C\left(\underline{a}_{0}, \bar{a}_{1}, \bar{a}_{2}, \underline{a}_{3}, \underline{a}_{4}, \ldots\right) \\
& A_{3}=C\left(\bar{a}_{0}, \underline{a}_{1}, \underline{a}_{2}, \bar{a}_{3}, \bar{a}_{4}, \ldots\right) \\
& A_{4}=C\left(\bar{a}_{0}, \bar{a}_{1}, \underline{a}_{2}, \underline{a}_{3}, \ldots\right),
\end{aligned}
$$

in analogy with the Kharitonov polynomials given by (4). The matrices $B_{1}, B_{2}, B_{3}, B_{4}$ are defined in the same manner for the family $\mathcal{B}$. We are now ready to state the main result of this subsection.

Theorem 3.1: Consider the interval matrix families $\mathcal{A}, \mathcal{B}$ given by (13), and assume that all the matrices belonging to $\mathcal{A}, \mathcal{B}$ are Hurwitz. Then for every pair of LTI systems of the form $\Sigma_{A}, \Sigma_{B}$ with $A \in \mathcal{A}, B \in \mathcal{B}$ to have a CQLF, it is necessary and sufficient that none of the eight matrix products

$$
\begin{aligned}
& A_{1} B_{2}, A_{1} B_{3}, A_{2} B_{1}, A_{2} B_{4}, \\
& A_{3} B_{1}, A_{3} B_{4}, A_{4} B_{2}, A_{4} B_{3},
\end{aligned}
$$

has a negative real eigenvalue.

Proof: Let $A=C\left(a_{0}, \ldots, a_{n-1}\right), B=C\left(b_{0}, \ldots, b_{n-1}\right)$ be two matrices in the families $\mathcal{A}$ and $\mathcal{B}$ respectively, and write $B=A-g h^{T}$ where $g=(0,0, \ldots, 1)^{T}$, and $h=$ $\left(b_{0}-a_{0}, \ldots, b_{n-1}-a_{n-1}\right)^{T}$. Then it follows from the Circle Criterion that the LTI systems $\Sigma_{A}, \Sigma_{B}$ have a CQLF if and only if the rational function

$$
1+h^{T}(s I-A)^{-1} g
$$

is strictly positive real.

It can be verified by direct computation that

$$
1+h^{T}(s I-A)^{-1} g=b(s) / a(s)
$$

where the polynomials $a(s), b(s)$ are given by

$$
\begin{aligned}
& a(s)=a_{0}+a_{1} s+\cdots a_{n-1} s^{n-1}+s^{n} \\
& b(s)=b_{0}+b_{1} s+\cdots b_{n-1} s^{n-1}+s^{n} .
\end{aligned}
$$

It now follows that every pair of LTI systems $\Sigma_{A}, \Sigma_{B}$ with $A \in \mathcal{A}, B \in \mathcal{B}$ will have a CQLF if and only if all of the rational functions $b(s) / a(s)$ are strictly positive real where $a(s)$ and $b(s)$ belong to the interval polynomial families

$$
\begin{aligned}
& a(s)=a_{0}+a_{1} s+\cdots a_{n-1} s^{n-1}+s^{n} \\
& \text { with } \underline{a}_{i} \leq a_{i} \leq \bar{a}_{i} \text { for } 0 \leq i \leq n-1
\end{aligned}
$$

and

$$
\begin{aligned}
& b(s)=b_{0}+b_{1} s+\cdots b_{n-1} s^{n-1}+s^{n} \\
& \text { with } \underline{b}_{i} \leq b_{i} \leq \bar{b}_{i} \text { for } 0 \leq i \leq n-1 .
\end{aligned}
$$

respectively. By a slight abuse of notation, we shall use the notation $\mathcal{A}, \mathcal{B}$ to denote these polynomial families also. 
Now, Theorem 2.3 establishes that all of the rational functions in $\mathcal{B} / \mathcal{A}$ are strictly positive real if and only if the functions

$$
\begin{aligned}
& \frac{k_{2}^{\mathcal{B}}(s)}{k_{1}^{\mathcal{A}}(s)}, \frac{k_{3}^{\mathcal{B}}(s)}{k_{1}^{\mathcal{A}}(s)}, \frac{k_{1}^{\mathcal{B}}(s)}{k_{2}^{\mathcal{A}}(s)}, \frac{k_{4}^{\mathcal{B}}(s)}{k_{2}^{\mathcal{A}}(s)}, \\
& \frac{k_{1}^{\mathcal{B}}(s)}{k_{3}^{\mathcal{A}}(s)}, \frac{k_{4}^{\mathcal{B}}(s)}{k_{3}^{\mathcal{A}}(s)}, \frac{k_{2}^{\mathcal{B}}(s)}{k_{4}^{\mathcal{A}}(s)}, \frac{k_{3}^{\mathcal{B}}(s)}{k_{4}^{\mathcal{A}}(s)},
\end{aligned}
$$

are strictly positive real. The result now follows from Theorem 2.1.

\section{Remarks:}

The above result provides simple conditions that are necessary and sufficient for CQLF existence for a pair of LTI systems in companion form subject to interval uncertainty. In fact, it is only necessary to calculate the eigenvalues of eight matrices and check that none are real and negative. On the other hand, testing via the strict positive real condition on the transfer functions requires checking that the functions are positive for infinitely many values of $\omega$.

\section{B. CQLF existence for general pairs of interval matrices differing by rank one}

We shall next consider a problem that is closely related to that dealt with in Theorem 3.1 above. Let $\mathcal{A}$ be a real interval matrix family in $\mathbb{R}^{n \times n}$ of the form

$$
\mathcal{A}=\left\{A \in \mathbb{R}^{n \times n}: \underline{a}_{i j} \leq a_{i j} \leq \bar{a}_{i j} \text { for } 1 \leq i, j \leq n\right\},
$$

and let $g, h$ be two fixed vectors in $\mathbb{R}^{n}$. Theorem 3.2 below is concerned with the following question.

Determine necessary and sufficient conditions for any pair of LTI systems $\Sigma_{A}, \Sigma_{A-g h^{T}}$ where $A \in$ $\mathcal{A}$ to have a CQLF.

It is important to note that in this situation, the interval uncertainty is in the matrix $A$, while the difference $g h^{T}$ is fixed. In the previous subsection, we considered two systems in companion form, each of which was independently subject to interval uncertainty.

As in the previous subsection, we shall assume that all of the matrices $A, A-g h^{T}$ for $A \in \mathcal{A}$ are Hurwitz. Also, in the statement of the next theorem, the notation $\mathcal{A}_{1}$ is used to denote the set of all matrices belonging to $\mathcal{A}$ where one entry $a_{i j}$ is set equal to one of the interval endpoints $\underline{a}_{i j}$ or $\bar{a}_{i j}$.

Theorem 3.2: Let $\mathcal{A}$ be a real interval matrix family of the form (17) and let $g, h$ be two fixed vectors in $\mathbb{R}^{n}$ such that all matrices $A, A-g h^{T}$ for $A \in \mathcal{A}$ are Hurwitz. Let $f\left(s, a_{i j}\right)$ be the polynomial in $n^{2}+1$ variables given by

$$
f\left(s, a_{i j}\right)=\operatorname{det}\left(s I-A\left(A-g h^{T}\right)\right) .
$$

Then the systems $\Sigma_{A}, \Sigma_{A-g h^{T}}$ have a CQLF for each $A$ in $\mathcal{A}$ if and only if: (i) there is no solution to the set of simultaneous equations

$$
\begin{aligned}
f\left(s, a_{i j}\right) & =0 \\
\frac{\partial f}{\partial a_{i j}}\left(s, a_{i j}\right) & =0
\end{aligned}
$$

in the range $-\infty<s<0, \underline{a}_{i j} \leq a_{i j} \leq \bar{a}_{i j}$;

(ii) for each $A$ in the reduced family $\mathcal{A}_{1}$, there is a CQLF for the systems $\Sigma_{A}, \Sigma_{A-g h^{T}}$.

Proof: First of all, Theorem 2.2 establishes that for fixed Hurwitz matrices $A, A-g h^{T}$, the systems $\Sigma_{A}, \Sigma_{A-g h^{T}}$ have a CQLF if and only if the matrix product $A\left(A-g h^{T}\right)$ has no negative real eigenvalues. Thus, $\Sigma_{A}, \Sigma_{A-g h^{T}}$ will have a CQLF for any $A$ in $\mathcal{A}$ if and only if $A\left(A-g h^{T}\right)$ has no negative real eigenvalues for all $A$ in $\mathcal{A}$. Equivalently, the polynomial $f\left(s, a_{i j}\right)$ must have the same sign for all $s<0$ and all $a_{i j}$ with $\underline{a}_{i j} \leq a_{i j} \leq \bar{a}_{i j}$. In fact, for $n$ even (odd) we must have $f\left(s, a_{i j}\right)>0\left(f\left(s, a_{i j}\right)<0\right)$ for all $s<0$, and $\underline{a}_{i j} \leq a_{i j} \leq \bar{a}_{i j}$. We shall prove the result for the case when $n$ is even. The proof for odd $n$ proceeds identically.

It follows from Theorem 2.4 that $f\left(s, a_{i j}\right)>0$ for all $s<0$, and $\underline{a}_{i j} \leq a_{i j} \leq \bar{a}_{i j}$ if and only if:

(i) there is no solution to the set of equations (18) within the range $-\infty<s<0, \underline{a}_{i j} \leq a_{i j} \leq \bar{a}_{i j}$;

(ii) all of the polynomials obtained by fixing the value of one of the variables $a_{k l}$ to one of the endpoints $\underline{a}_{k l}$ or $\bar{a}_{k l}$ are positive within the allowed ranges for the remaining variables.

Condition (i) above is obviously equivalent to condition (i) in the statement of the theorem while condition (ii) is equivalent to requiring that for all matrices $A$ belonging to the family $\mathcal{A}_{1}$, the product $A\left(A-g h^{T}\right)$ has no negative real eigenvalues, or equivalently by Theorem 2.2 that the systems $\Sigma_{A}, \Sigma_{A-g h^{T}}$ have a CQLF. This completes the proof.

\section{Remarks:}

(i) Condition (ii) of Theorem 3.2 replaces the original problem with a finite number of CQLF existence problems for interval matrix families. However, the number of uncertain parameters in each of these problems has been reduced by one, as one of the entries $a_{i j}$ is fixed in each case. Repeatedly applying the theorem to these reduced problems will eventually lead to a finite number of fixed coefficient CQLF existence problems, each of which can be solved using Theorem 2.2.

(ii) Condition (i) involves $n^{2}+1$ polynomial equations in the $n^{2}+1$ variables $s, a_{i j}, 1 \leq i, j \leq n$. While this condition is difficult to check in general, for some classes of system it may be possible to verify that it is automatically satisfied, thus reducing the problem of CQLF existence under interval uncertainty to a number 
of fixed coefficient CQLF existence problems. We shall next give an example where this is indeed the case.

Corollary 3.1: Let $\mathcal{A}$ be an interval matrix family in companion form in $\mathbb{R}^{2 \times 2}$ consisting of matrices of the form

$$
A=\left(\begin{array}{cc}
0 & 1 \\
-a_{1} & -a_{2}
\end{array}\right)
$$

where $\underline{a}_{i} \leq a_{i} \leq \bar{a}_{i}$ for $i=1,2$, and let $g=(0,1)^{T}$ and $h=\left(h_{1}, h_{2}\right)^{T}$ be fixed. Moreover, assume that for every $A \in \mathcal{A}, A$ and $A-g h^{T}$ are both Hurwitz. Then, for every $A \in \mathcal{A}$, the LTI systems $\Sigma_{A}, \Sigma_{A-g h^{T}}$ have a CQLF if and only if $\Sigma_{A_{i}}, \Sigma_{A_{i}-g h^{T}}$ have a CQLF for $1 \leq i \leq 4$, where $A_{1}, \ldots, A_{4}$ are the four fixed coefficient matrices obtained by setting $a_{i}$ equal to $\underline{a}_{i}$ or $\bar{a}_{i}$ for $i=1,2$.

Proof: From Theorem 3.2, it is enough to show that there can be no solution to the equations (18) in the range $\underline{a}_{i} \leq$ $a_{i} \leq \bar{a}_{i},-\infty<s<0$. By assumption, all matrices $A$, $A-g h^{T}$ for $A \in \mathcal{A}$ are Hurwitz. It follows from this that for each $A$ belonging to $\mathcal{A}, a_{i}>0$ and $a_{i}+h_{i}>0$ for $i=1,2$.

Now the polynomial $f\left(s, a_{i j}\right)=\operatorname{det}\left(s I-A\left(A-g h^{T}\right)\right)$ is given by

$f\left(s, a_{i j}\right)=s^{2}+s\left(2 a_{1}+h_{1}-a_{2}\left(a_{2}+h_{2}\right)\right)+a_{1}\left(a_{1}+h_{1}\right)$.

Thus, in the current situation, condition (18) requires that

$$
\begin{aligned}
f\left(s, a_{1}, a_{2}\right) & =0 \\
\frac{\partial f}{\partial a_{1}}=2 s+2 a_{1}+h_{1} & =0 \\
\frac{\partial f}{\partial a_{2}}=-s\left(2 a_{2}+h_{2}\right) & =0 .
\end{aligned}
$$

But as $a_{2}>0$ and $a_{2}+h_{2}>0$, the only possible solution to the third equation in (20) under the hypotheses of the corollary is $s=0$. The result now follows immediately.

\section{Application}

In this section we present a numerical example to illustrate the use of Theorem 3.1. Consider the two interval families of companion matrices, $\mathcal{A}, \mathcal{B}$ (corresponding to the rational transfer function families in [29]), in $\mathbb{R}^{3 \times 3}$ given by

$$
\begin{aligned}
& \mathcal{A}=\left\{C\left(a_{0}, a_{1}, a_{2}\right): a_{0} \in[1,2], a_{1} \in[5,6], a_{2} \in[3,4]\right\}, \\
& \mathcal{B}=\left\{C\left(b_{0}, b_{1}, b_{2}\right): b_{0} \in[1,1], b_{1} \in[1,2], b_{2} \in[3,4]\right\},
\end{aligned}
$$

where, as before, we use the notation $C\left(a_{0}, a_{1}, a_{2}\right)$ to denote the companion matrix whose last row is $\left(-a_{0},-a_{1},-a_{2}\right)$. Then, both $\mathcal{A}$ and $\mathcal{B}$ consist of Hurwitz matrices and the corresponding Kharitonov family of companion matrices defined by (14) are as follows

$$
\begin{aligned}
& A_{1}=C(1,5,4), A_{2}=C(1,6,4) \\
& A_{3}=C(2,5,3), A_{4}=C(2,6,3) \\
& B_{1}=C(1,1,4), B_{2}=C(1,2,4) \\
& B_{3}=C(1,1,3), B_{4}=C(1,2,3)
\end{aligned}
$$

Now one can compute the eight matrix products of Theorem 3.1 and check the corresponding eigenvalues. The calculated eigenvalues of the matrix products are presented in the figure below.

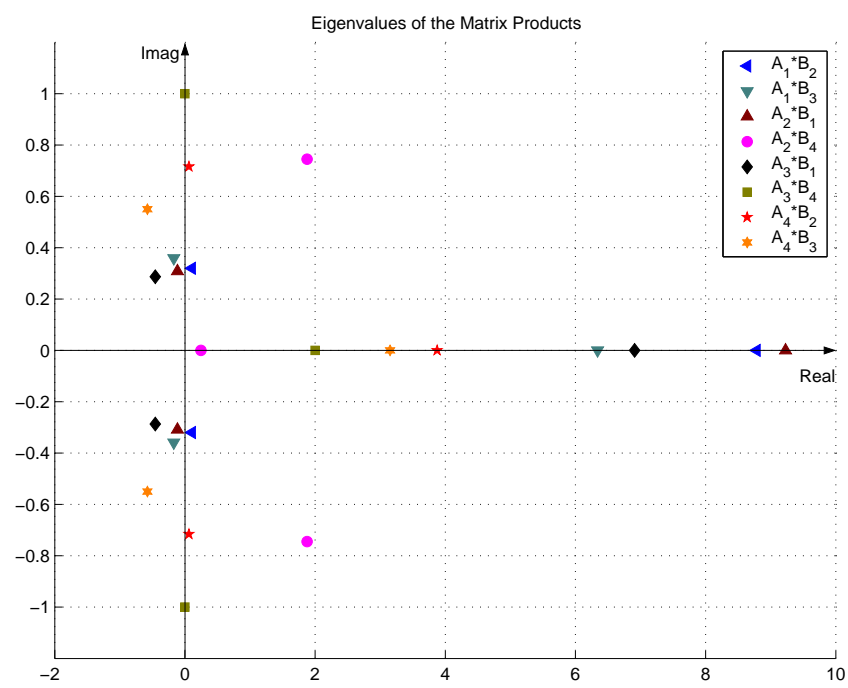

As can be seen from the plot none of the matrix products have negative real eigenvalues. Theorem 3.1 therefore guarantees the existence of a CQLF for any pair of LTI systems $\Sigma_{A}, \Sigma_{B}$ where $A \in \mathcal{A}, B \in \mathcal{B}$.

\section{CONCLUDING REMARKS}

In this paper, we have considered the stability question for switched linear systems subject to interval uncertainty. In particular, we have presented two results on common quadratic Lyapunov function existence for pairs of LTI systems with interval uncertainty in the entries of their system matrices. These results provide verifiable conditions for CQLF existence for interval matrix families in companion form. A numerical example has been presented to illustrate how the results of the paper can be used in practice.

Acknowledgements: This work was partially supported by Science Foundation Ireland grant 00/PI.1/C067 and Science Foundation Ireland Basic Research Grant 04/BR/m0061. Science Foundation Ireland is not responsible for any use of data appearing in this publication.

\section{REFERENCES}

[1] K. S. Narendra and J. H. Taylor, Frequency Domain Criteria for Absolute Stability. Academic Press, 1973.

[2] D. Liberzon, Switching in systems and control. Birkhauser, 2003.

[3] D. Liberzon and A. S. Morse, "Basic problems in stability and design of switched systems," IEEE Control Systems Magazine, vol. 19, no. 5, pp. 59-70, 1999.

[4] N. Cohen and I. Lewkowicz, "A necessary and suffi cient criterion for the stability of a convex set of matrices," IEEE Transactions on Automatic Control, vol. 38, no. 4, pp. 611-615, 1993.

[5] T. Ando, "Sets of matrices with a common Lyapunov solution," Archiv der Mathematik, vol. 77, pp. 76-84, 2001. 
[6] D. Cheng, L. Guo, and J. Huang, "On quadratic Lyapunov functions," IEEE Transactions on Automatic Control, vol. 48, no. 5, pp. 885-890, 2003.

[7] R. N. Shorten and K. S. Narendra, "On common quadratic Lyapunov functions for pairs of stable LTI systems whose system matrices are in companion form," IEEE Transactions on Automatic Control, vol. 48, no. 4, pp. 618-621, 2003.

[8] R. N. Shorten, K. S. Narendra, and O. Mason, "A result on common quadratic Lyapunov functions," IEEE Transactions on Automatic Control, vol. 48, no. 1, pp. 110-113, 2003.

[9] R. N. Shorten and K. S. Narendra, "Necessary and suffi cient conditions for the existence of a common quadratic Lyapunov function for a fi nite number of stable second order linear time-invariant systems," International Journal of Adaptive Control and Signal Processing, vol. 16, pp. 709-728, 2003.

[10] N. Cohen and I. Lewkowicz, "Convex invertible cones and the Lyapunov equation," Linear Algebra and its Applications, vol. 250, no. 1, pp. 105-131, 1997.

[11] N. Cohen, I. Lewkowicz, and L. Rodman, "Exponential stability of triangular differential inclusion systems," Systems \& Control Letters, vol. 30, pp. 159-164, 1997.

[12] N. Cohen and I. Lewkowicz, "A pair of matrices sharing common Lyapunov solutions - a closer look," Linear Algebra and its Applications, vol. 360, pp. 83-104, 2003.

[13] N. Cohen and I. Lewkowicz, "Convex invertible cones of state space systems," Mathematics and Control of Signals and Systems, vol. 10, pp. 265-286, 1997.

[14] D. Hershkowitz, "On cones and stability," Linear algebra and its applications, vol. 275-276, pp. 249-259, 1998.

[15] D. Hinrichsen and A. J. Pritchard, "Real and complex stability radii: A survey," in Control of Uncertain Systems, pp. 119-162, Birkhauser, 1989.

[16] R. E. Kalman, "Lyapunov functions for the problem of Lur'e in automatic control," Proceedings of the National Academy of Sciences, vol. 49, no. 2, pp. 201-205, 1963.

[17] I. Lewkowicz, "A necessary condition for quantitative exponential stability of state spacesystems," Systems \& Control Letters, vol. 38, pp. 1-4, 1999.

[18] R. Loewy, "On ranges of real Lyapunov transformations," Linear Algebra and its Applications, vol. 13, pp. 79-89, 1976.

[19] K. S. Narendra and J. Balakrishnan, "A common Lyapunov function for stable LTI systems with commuting $\mathcal{A}$-matrices," IEEE Transactions on automatic control, vol. 39, no. 12, pp. 2469-2471, 1994.

[20] T. Ooba and Y. Funahashi, "Two conditions concerning common quadratic Lyapunov functions for linear systems," IEEE Transactions on Automatic Control, vol. 42, no. 5, pp. 719-721, 1997

[21] T. Ooba and Y. Funahashi, "On a common quadratic Lyapunov function for widely distant systems," IEEE Transactions on Automatic Control, vol. 42, no. 12, pp. 1697-1699, 1997.

[22] R. N. Shorten, O. Mason, F. O. Cairbre, and P. Curran, "A unifying framework for the SISO Circle Criterion and other quadratic stability criteria," International Journal of Control, vol. 77, no. 1, pp. 1-8, 2004.

[23] J. C. Willems, "Least squares stationary optimal control and the algebraic Ricatti equation," IEEE Trans. on Automatic Control, vol. 16 , no. 6, pp. 621-634, 1971.

[24] J. C. Willems, "Dissipative dynamical systems, Part I and II," Arch. Rational Mech. Anal., vol. 45, pp. 321-351, 352-393, 1972.

[25] C. King and R. Shorten, "A spectral condition for the existence of a CQLF," in American Control Conference, 2004.

[26] K. S. Narendra and R. M. Goldwyn, "A geometrical criterion for the stability of certain non-linear non-autonomous systems," IEEE Transactions on Circuit Theory, vol. 11, no. 3, pp. 406-407, 1964.

[27] K. Meyer, "On the existence of Lyapunov functions for the problem of Lur'e," J. SIAM Control, vol. 3, no. 3, pp. 373-383, 1966.

[28] R. N. Shorten, P. Curran, and K. Wulff, "On time-domain multiplier criteria for single-input single-output systems," International Journal of Control, vol. 77, no. 11, pp. 985-991, 2004.

[29] H. Chapellat, M. Dahleh, and S. Bhattacharyya, "On robust nonlinear stability of interval control systems," IEEE Transactions on Automatic Control, vol. 36, no. 1, pp. 59-67, 1991.

[30] E. Walach and E. Zeheb, "Sign test of multivariable real polynomials," IEEE Transactions on Circuits and Systems, vol. 27, no. 7, pp. $619-625,1980$
[31] M. Sezer and D. Siljak, "On stability of interval matrices," IEEE Transactions on Automatic Control, vol. 39, no. 2, pp. 368-371, 1994.

[32] J. Rojas and J. Collada, "Stability of interval matrices using the distance to the set of unstable matrices," in Proc. of the American Control Conference, (Maryland), pp. 238-239, 1994.

[33] E. Zeheb, "Necessary and suffi cient conditions for robust stability of a continuous system-the continuous dependency case illustrated via multilinear dependency," IEEE Trans. on Circuits and Systems, vol. 37, no. 1, pp. 47-53, 1990 .

[34] O. Mason, Switched systems, convex cones and common Lyapunov functions. $\mathrm{PhD}$ thesis, Department of Electronic Engineering, National University of Ireland, Maynooth, 2004.

[35] T. Kailath, Linear systems. Prentice Hall, New Jersey, 1980.

\section{APPENDIX}

\section{Proof of Theorem 2.1:}

Without loss of generality, we may assume that $g h^{T}$ is in one the Jordan canonical forms

$$
\begin{aligned}
& \text { (i) }\left(\begin{array}{cccc}
c & 0 & \ldots & 0 \\
0 & \ldots & \ldots & 0 \\
\vdots & & & \\
0 & \ldots & \ldots & 0
\end{array}\right) \text {, } \\
& \text { (ii) }\left(\begin{array}{cccc}
0 & \ldots & \ldots & 0 \\
1 & \ldots & \ldots & 0 \\
\vdots & & & \\
0 & \ldots & \ldots & 0
\end{array}\right) \text {. }
\end{aligned}
$$

As $A$ and $A-g h^{T}$ are both Hurwitz, their determinants will have the same sign, so it follows that the product $A(A-$ $\left.g h^{T}\right)$ has no negative real eigenvalues if and only if, for all $\lambda>0$

$\operatorname{det}\left(\lambda I+\left(A-g h^{T}\right) A\right)=\operatorname{det}\left(\lambda I+A^{2}-g h^{T} A\right)>0$

If $g h^{T}$ is in Jordan form then it follows that the expressions

$$
\operatorname{det}\left(\lambda I+A^{2}-g h^{T} A\right)
$$

and

$$
\operatorname{Re}\left\{\operatorname{det}\left(\lambda I+A^{2}-g h^{T} A-\sqrt{\lambda} j g h^{T}\right)\right\},
$$

are identical. Thus, writing $\lambda=\omega^{2}$ we have that for all real $\omega$

$$
\operatorname{Re}\left\{\operatorname{det}\left(\omega^{2} I+A^{2}-g h^{T} A-j \omega g h^{T}\right)\right\}>0 .
$$

It now follows, after a short calculation ([34]) that for all $\omega \in \mathbb{R}$

$1+\operatorname{Re}\left\{\frac{\operatorname{det}\left(j \omega I-\left(A-g h^{T}\right)\right)-\operatorname{det}(j \omega I-A)}{\operatorname{det}(j \omega I-A)}\right\}>0$

and hence from the identity [35]

$\operatorname{det}\left(h^{T}(s I-A)^{-1} g\right)=\frac{\operatorname{det}\left(s I-\left(A-g h^{T}\right)\right)-\operatorname{det}(s I-A)}{\operatorname{det}(s I-A)}$

that for it follows that for all real $\omega$

$$
1+R e\left\{h^{T}(j \omega I-A)^{-1} g\right\}>0
$$

as claimed. 